“C 2007 IEEE. Personal use of this material is permitted. Permission from IEEE must be obtained for all other uses, in any current or future media, including reprinting/republishing this material for advertising or promotional purposes, creating new collective works, for resale or redistribution to servers or lists, or reuse of any copyrighted component of this work in other works." 


\section{HTS Levitated Mobile Technology and Prototype}

\author{
Jianxun Jin and Luhai Zheng \\ Center of Applied Superconductivity and Electrical Engineering \\ University of Electronic Science and Technology of China \\ ChengDu, SiChuan Province, 610054 China \\ jxjin@uestc.edu.cn
}

\author{
Youguang Guo, Jiaxin Chen and Jianguo Zhu \\ Faculty of Engineering \\ University of Technology \\ Sydney, NSW 2007, Australia \\ youguang@eng.uts.edu.au
}

\begin{abstract}
The special reaction phenomenon of a high temperature superconductor (HTS) to magnetic fields provides an alternative technical solution for design of a levitated mobilization system, in which a linear motion drive with a HTS incorporated is a critical issue. This work presents the design and performance analysis of a HTS linear synchronous driving system with a prototype built for verification. Magnetic field finite element analyses are conducted to compute the key parameters, and the steady state characteristic of the system is predicted by using the classic phasor voltage equation. A simulation model is also built to analyze the system dynamic performance, and results show that the levitated HTS linear motion system developed can be driven and controlled at the desired speed.
\end{abstract}

Index Terms - High temperature superconductor (HTS), HTS levitation, magnetic field, maglev, linear motor.

\section{INTRODUCTION}

Linear motor is a kind of driving equipment for converting electrical energy into linear movement directly without any inbetween transferring organization, and has advantages such as simple structure, no abrasion, low noise, high accuracy, and easy to maintain. After a long-term exploration and experimental stages, linear motor has entered the practical commodity stage since the 1970's. The appearing of new kinds of magnetic material, control technology, and coolant method, create a solid condition of linear motor for the economical application with high speed and high power. In 1990s, the low temperature superconductor (LTS) technology was applied to the linear synchronous motor (LSM) as to be the drive power of a maglev train in Japan, which increased speed significantly. Since the discovery of high temperature superconductor (HTS) material [1], the HTS linear motor technology has soon developed and obtained a fast development as well as a great progress on the HTS applied to conventional rotating machine and many other fields [2]-[10].

Now, the developed HTS linear motors mainly in the form of LSM, include the (i) HTS bulks used in moving secondary [11]-[14], and (ii) the HTS coils applied in the static primary [15] and can be subdivided into single-side [11],[15] and double-side [12]-[14] in structures. Because stable levitation force can be generated by a permanent magnet (PM) like NdFeB over a YBaCuO HTS bulk [16], HTS levitation system contained with PMs and YBCuO bulks can be used to develop a HTS linear driving system. The main advantage of the HTS levitation is its strong levitating force with passive and selfstabilizing feature [17],[18], leading to the removal of the sophisticated control system for regulating the air gap between guideway and levitated moving secondary, which is necessary for other types of levitation transportation systems. So that the levitation system integrated with HTS are suitable to be applied in a maglev system for example, and many other transportations and mobilizations.

Compared to the conventional linear motors, HTS linear motors have a number of obvious advantages, such as smaller volume, less weight, bigger propulsion, and higher power factor. This paper presents the development of a HTS LSM driving system with a small-scale prototype HTS levitated mobile. Both the working principles of the linear motor for driving and the PM-HTS bulks for lifting the mobile are described. For design and performance analysis of the HTS LSM, magnetic field finite element analysis (FEA) is carried out for accurately calculating the major parameters such as winding flux, back electromotive force (emf), winding inductance, and cogging torque. The steady state characteristic of the motor is predicted by using the classic phasor voltage equation, which can provide a reasonable result if the fundamental components of the applied voltage, back emf and current are dominant. The classic phasor voltage equation is used to predict the motor's steady state characteristic under the optimal brushless dc control (BLDC) condition, i.e. the back emf is in phase with the stator current. A Matlab/Simulinkbased model, capable of taking into account the real curves of key motor parameters against mover position, is compiled to predict effectively the dynamic drive performance under a BLDC control scheme.

\section{Prototype And Maglev Force}

Fig. 1 illustrates the scheme of the HTS prototype levitator propelled by a HTS LSM. The primary and the secondary of the HTS linear motor are composed of $3 \Phi$ copper windings and HTS bulk magnets, respectively. The levitated mobile is lifted by a levitation system consisting of field-cooled HTS bulks on the mobile bottom and PMs placed on the guideway along the mobile movement direction. As shown in the Fig. 1, PMs, the same pole in longitudinal direction and alternating poles of $\mathrm{N}$ and $\mathrm{S}$ in transverse direction, are located at both sides of the primary windings to levitate and guide the mobile. While the levitating force is directly proportional to the gradient of magnetic field over the guideway, the lateral guiding force depends on the trapped flux in the HTS bulks. The HTS levitation system has inherent stability in both vertical and lateral directions, realizes selflevitation and self-guidance without any active control system. 
The levitation force and guidance force are generated based on the pinning force or the shielding force of the HTS bulk [17]-[19]. The magnetic levitation force and guidance force between a HTS bulk and PM are modelled as Fig. 2 and illustrated as Fig. 3. When the materials and dimensions of the PM-HTS bulks are properly chosen, an appropriate levitation force and guidance force can be generated for levitating and guiding the designed prototype.

The levitation force and guidance force between a PM and an HTS bulk can be calculated based on the Lorentz force $F$, which is related to current density $J$ as

$$
F=J \times B
$$

Then the HTS-PM levitation force $F_{L e v}$ and guidance force $F_{G u i}$ are given by

$$
\begin{aligned}
& F_{L e v}(x, z)=\iiint_{V_{S C}} J_{y}\left(x^{\prime}, z^{\prime}\right) \times B_{x}\left(x^{\prime}, z^{\prime}\right) d v \\
& F_{G u i}(x, z)=\iiint_{V_{S C}} J_{y}\left(x^{\prime}, z^{\prime}\right) \times B_{z}\left(x^{\prime}, z^{\prime}\right) d v
\end{aligned}
$$

where $v$ is volume, $V_{S C}$ is the HTS volume, $J_{y}\left(x^{\prime}, z^{\prime}\right)$ is the superconducting current density distribution at $\left(\mathrm{x}^{\prime}, \mathrm{z}^{\prime}\right)$ in the superconductor and in the y direction of Fig. $1, B_{x}\left(x^{\prime}, z^{\prime}\right)$ is the magnetic flux density generated by the PM in the $\mathrm{x}$ direction at $\left(x^{\prime}, z^{\prime}\right)$ without superconductor applied, $B_{z}\left(x^{\prime}, z^{\prime}\right)$ is the PM magnetic flux density in the $\mathrm{z}$ direction at $\left(x^{\prime}, z^{\prime}\right)$ without superconductor applied. To solve the above equation, it is necessary to know the applied field $B_{x}\left(x^{\prime}, z^{\prime}\right)$ and $B_{z}\left(x^{\prime}, z^{\prime}\right)$, and the current distribution $J_{y}\left(x^{\prime}, z^{\prime}\right)$.

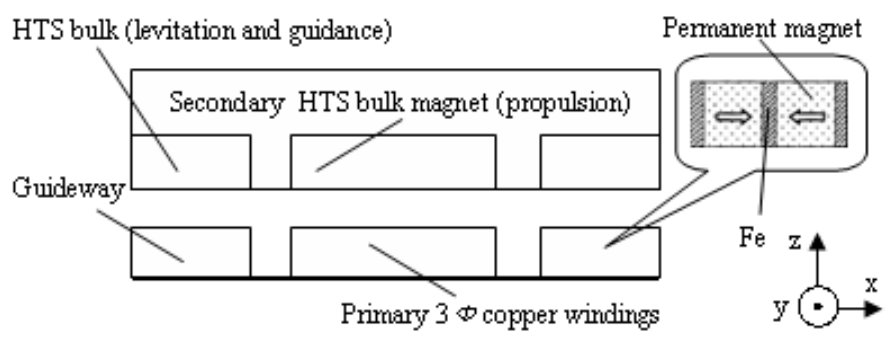

Fig. 1 Schematic diagram of an HTS maglev vehicle prototype.

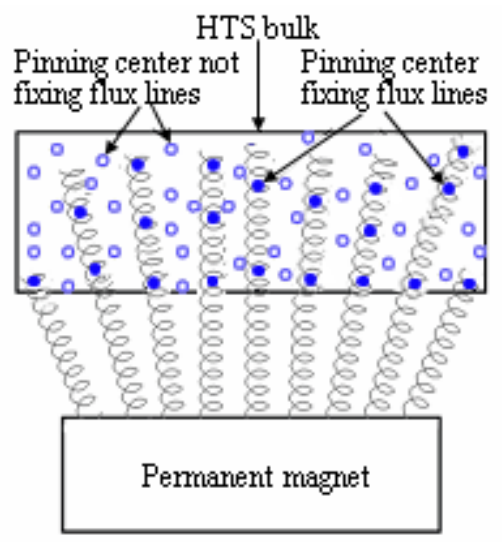

Fig. 2 Illustration of HTS levitation theoretical model [17].

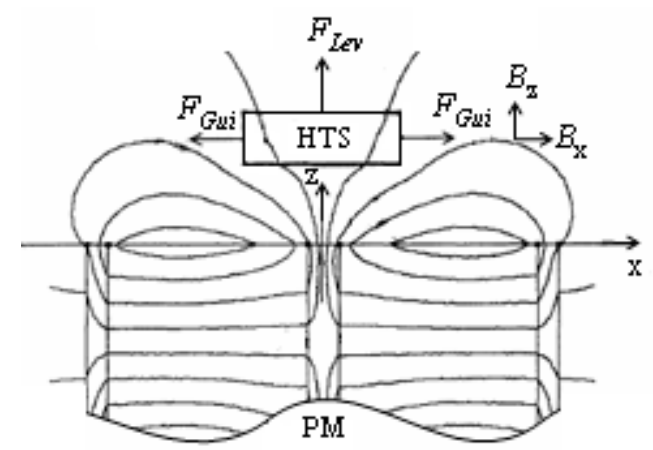

Fig. 3 Illustration of interaction between HTS bulk and PM.

\section{LINEAR MOTOR DRIVE}

While the levitation force and guidance force are produced by the PM-HTS bulks, the driving force is produced by the interaction of magnetic fields of the bulk superconductor (BSC) magnets mounted on the mover sides and the currents flowing in the coils placed in the slots of stationary sidetracks. The prototype HTS linear motion driving system can be seen in Fig. 4, and Table 1 lists its major dimensions and parameters.

The thrust force vector $d F$, acting on an infinitely thin stator conductor element vector $d s$ carrying stator current $I_{\mathrm{s}}$, is given by

$$
d F=I_{s} d s \times B
$$

If the stator conductor cannot be considered as infinitely thin and the flux density vector $B$ is averaged with respect to the axis y, then (4) leaded to

$$
d F=J_{s} \mathrm{Ye}_{\mathrm{s}} \times B_{a v} d A
$$

where $B_{a v}$ is the flux density vector averaged with respect to the axis $\mathrm{y}, J_{\mathrm{s}}$ is the current density in the stator conductor, $Y$ is the conductor length in the $\mathrm{y}$ direction, $e_{\mathrm{s}}$ is the unity vector pointing in the direction of flowing current in the stator conductor, and $d A$ is a differential element of the stator conductor cross section. The total force vector $F$ produced by two poles of the HTS linear drive can be calculated by integration over cross sections of all stator conductors.

TABLE I

MAJOR PARAMETERS AND DIMENSIONS OF THE HTS LINEAR MOTOR

\begin{tabular}{|l|l|}
\hline Dimensions and parameters & Quantities \\
\hline BSC magnet length (magnetization direction) $(\mathrm{mm})$ & 20 \\
\hline BSC magnet width (movement direction) $(\mathrm{mm})$ & 30 \\
\hline BSC magnet height $(\mathrm{mm})$ & 10 \\
\hline Gap between adjacent BSC magnets $(\mathrm{mm})$ & 10 \\
\hline Thickness of bulk back iron $(\mathrm{mm})$ & 5 \\
\hline Tooth length $(\mathrm{mm})$ & 5 \\
\hline Tooth width $(\mathrm{mm})$ & 10 \\
\hline Tooth depth $(\mathrm{mm})$ & 10 \\
\hline Slot width $(\mathrm{mm})$ & 10 \\
\hline Main air gap $(\mathrm{mm})$ & 5 \\
\hline Number of turns of stator winding & 35 \\
\hline Diameter of copper wire $(\mathrm{mm})$ & 0.75 \\
\hline Resistance per phase $(\Omega)$ & 0.41 \\
\hline
\end{tabular}




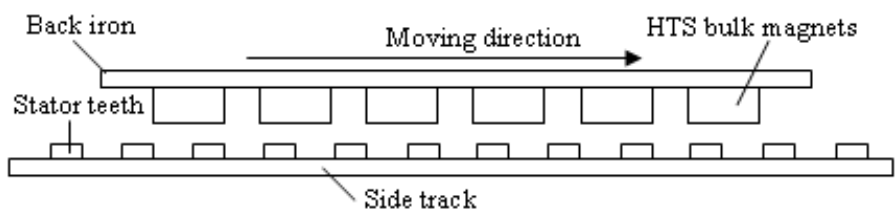

Fig. 4 Top view of the HTS linear motor.

The coils wound around stator teeth are connected to form 3 phase windings, which are supplied by a standard 3-phase inverter. It can be seen that only the windings facing the moving secondary would produce the driving force, while the others only cause additional resistance, power loss, and flux leakage. To deal with this problem, the windings can be divided into sections. The inverter is only connected to the active sections [20].

\section{Magnetic Field Fea And Parameter Calculation}

Magnetic field FEA can account for the detailed linear motor structure and the nonlinear properties of ferromagnetic materials and hence can accurately compute the field distribution and motor parameters. In this paper, twodimensional field computation in one pole-pair region is carried out, which can be sufficient for theoretical analysis. It should be noted that three-dimensional analysis within the whole motor could be conducted for considering the fringing effect and non-symmetry among three phases, at the expense of longer computational time.

The waveform of BSC magnet flux, defined as the winding flux produced by the mover BSC magnets, can be obtained from the no-load field solutions at different mover positions. To increase the computational accuracy and efficiency, the stator and mover are meshed separately from the middle of the air gap. When the mover moves to a specified position, the meshes are stitched along the middle air gap line. Periodical boundary conditions are applied. Fig. 5 shows the BSC magnet flux versus the mover position, which is almost sinusoidal.

When the mover moves, an emf is induced in the stator windings. The magnitude of fundamental flux component is computed as $\phi_{1}=0.0948 \mathrm{mWb}$, so the back emf and force constants, $K_{E}$ and $K_{F}$, can be determined as $1.47 \mathrm{Vs} / \mathrm{m}$ and 4.41 N/A respectively by

$$
\begin{gathered}
K_{E}=\pi N_{e} N_{s} \phi_{1} /(\tau \sqrt{ } 2) \\
K_{F}=m K_{E}
\end{gathered}
$$

where $N_{e}=6$ is the number of active coils per phase, $N_{s}$ the number of turns of a coil, $\tau$ the pole pitch and $m=3$ the number of phases.

By using a modified incremental energy method [21], the self and mutual inductances of the three phase windings are computed as 0.163 and $0.062 \mathrm{mH}$, respectively. Due to the large effective air gap in the surface mounted linear motor, the inductances remain constant at different mover positions.

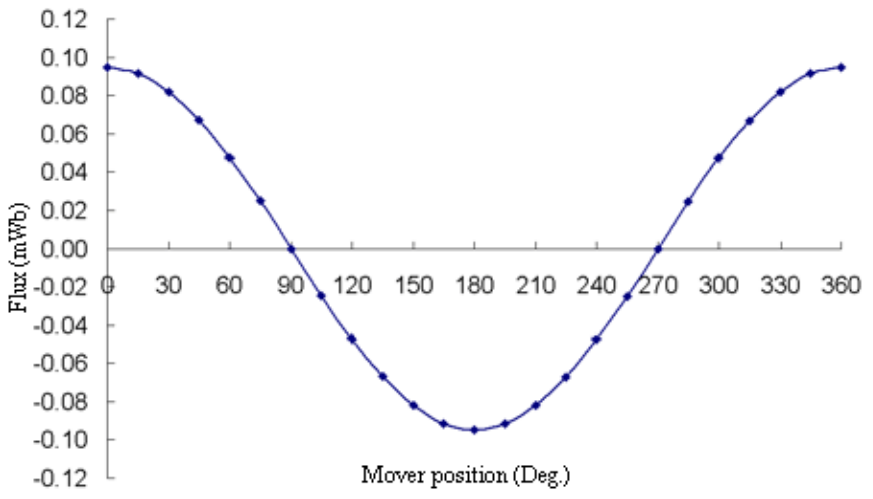

Fig. 5 BSC magnetic flux of one turn of one coil.

The cogging force can be obtained from the no-load field solutions by the Virtual work or the Maxwell stress tensor method. It is found that the cogging force is negligible.

\section{STEADY-STATE CHARACTERISTIC}

When the HTS linear drive operates in synchronous mode, its steady-state characteristic can be predicted by the phasor equation

$$
V_{1}=E_{1}+R_{1} I_{1}+j \omega_{1} L_{1} I_{1}
$$

where $V_{1}, E_{1}$ and $I_{1}$ are rms values of the applied voltage, the induced emf, and the current of a phase winding, respectively, $R_{1}$ is the phase winding resistance, $\omega_{1}$ is the angular frequency, and $L_{1}$ is the synchronous inductance which equals the selfinductance plus half mutual inductance here.

The LSM is assumed to operate in the optimum BSDC mode, i.e. $I_{1}$ in phase with $E_{1}$, so that the electromagnetic power and force can be calculated by

$$
\begin{gathered}
P_{e m}=m E_{1} I_{1} \\
F_{e m}=P_{e m} / v=K_{F} I_{1}
\end{gathered}
$$

where $v$ is the mover speed, and $E_{1}=K_{E} v$.

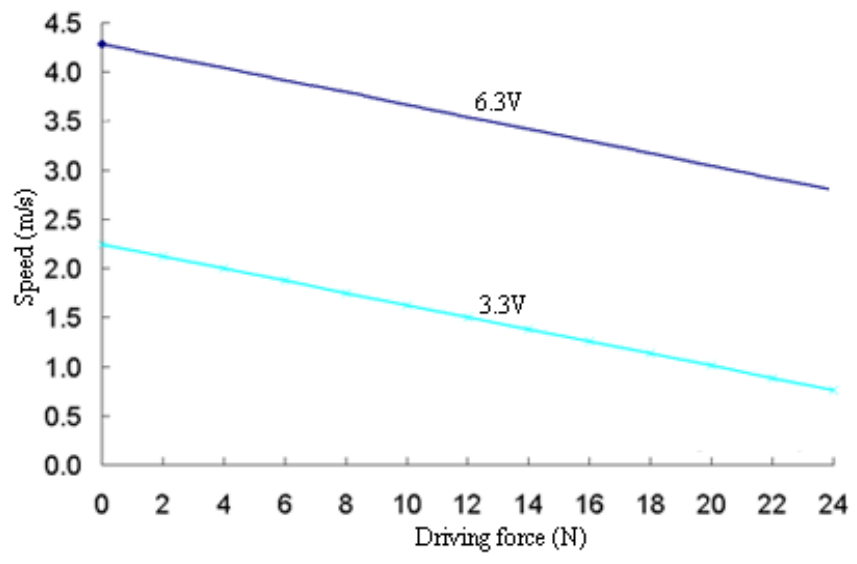

Fig. 6 Steady-state mechanical characteristics of the prototype. 
For a given terminal voltage $V_{1}$, the relationship between the mover speed and electromagnetic force is determined by (11)

$$
v=\frac{\sqrt{\left(\frac{R_{1} F_{e m}}{m}\right)^{2}+\left[\left(\frac{\pi L_{1} F_{e m}}{\tau K_{T}}\right)^{2}+K_{E}^{2}\right]\left[V_{1}^{2}-\left(\frac{R_{1} F_{e m}}{K_{T}}\right)^{2}\right]}-\frac{R_{1} F_{e m}}{m}}{\left(\frac{\pi L_{1} F_{e m}}{\tau K_{T}}\right)^{2}+K_{E}^{2}}
$$

The HTS levitator steady-state mechanical characteristics with different phase voltages are illustrated in Fig. 6.

\section{Performance Analysis By Phase Variable Model}

The dynamic performance of the linear motor under BLDC control can be analyzed by the following equationbased phase variable model [22],[23]

$$
\begin{gathered}
V_{k}=r_{k} i_{k}+d \lambda_{k} / d t+e_{k}, \quad k=a, b, c \\
\lambda_{k}=\sum_{q=a}^{c} L_{k q} i_{q} \\
F_{e m}=\frac{e_{a} i_{a}+e_{b} i_{b}+e_{c} i_{c}}{v}+F_{c o g}
\end{gathered}
$$

$$
m \frac{d v}{d t}=F_{e m}-B v-F_{L}
$$

All above variables are used as their conventional meanings [23]. The profiles of $L, e$ and $F_{c o g}$ can be obtained from a series of nonlinear FEA solutions, in which the mover position dependence and saturation effect are considered.

Suppose the electrical potentials of terminals $a, b, c$ and $N$-the neutral point of the Y-connected three phase windings are $U_{a}, U_{b}, U_{c}$ and $U_{N}$, respectively, one can obtain

$$
V_{k}=U_{k}-U_{N}, \quad k=a, b, c
$$

Assuming that the hard switching is applied, at the moment when phase $a$ is positively excited and phase $b$ is negatively excited, the following equations can be obtained

$$
U_{N}= \begin{cases}\frac{1}{3} \sum_{k=a}^{c}\left(U_{k}-E_{k}\right) & i_{c} \neq 0 \\ \frac{1}{2} \sum_{k=a}^{b}\left(U_{k}-E_{k}\right) & i_{c}=0\end{cases}
$$

According to (12)-(17), a Matlab/Simulink-based simulation model, as shown in Fig. 7, can be built to analyze the HTS linear drive's dynamic performance.

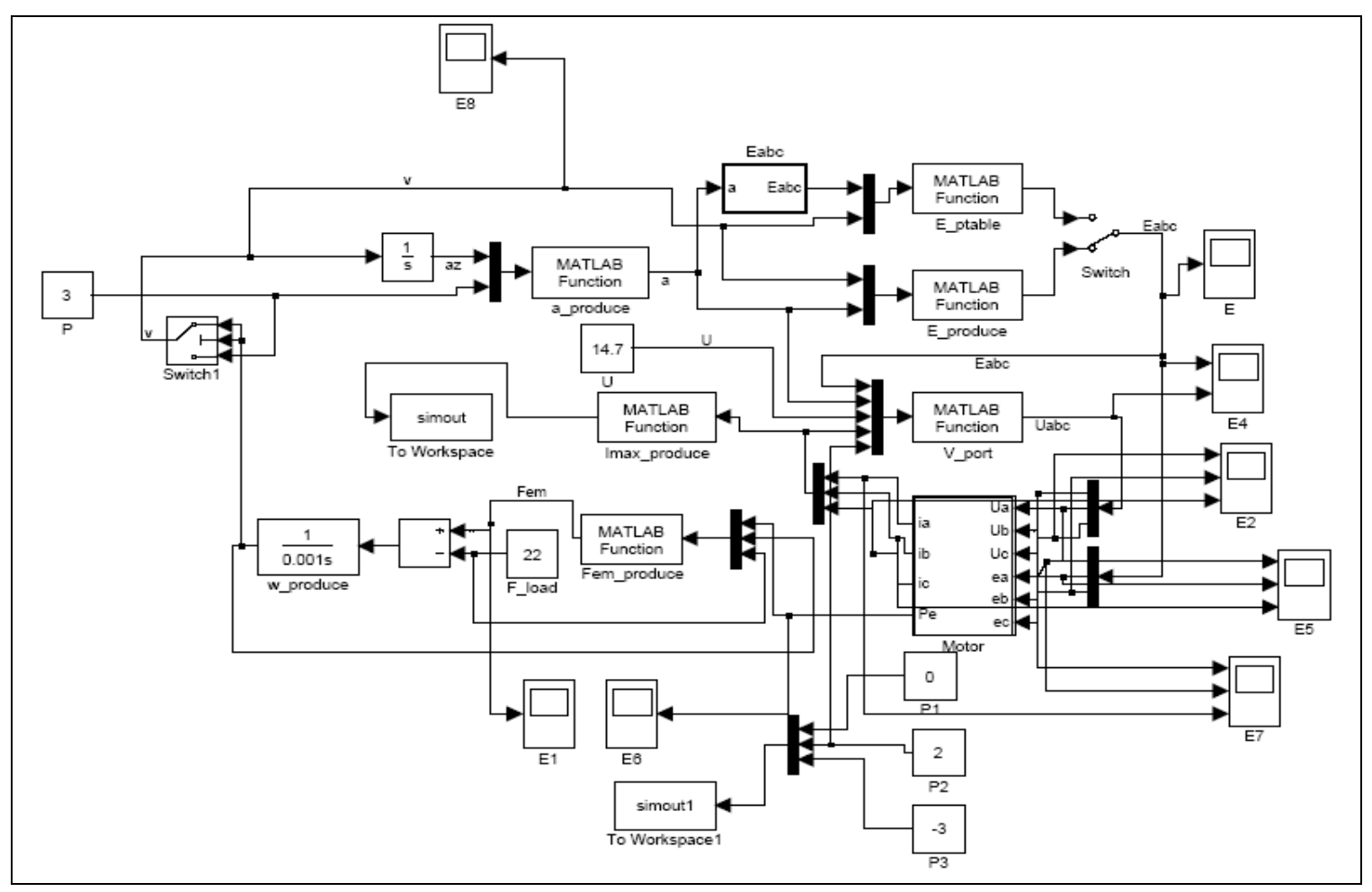

Fig. 7 Matlab/Simulink-based simulation model of the BSCDC motor with sinusoidal back emf.

As an example, Fig. 8 shows the start-up speed curve of the HTS LSM with full load when the rated voltage of the inverter is applied. The developed prototype can drive the levitated mobile at the rated speed of $3 \mathrm{~m} / \mathrm{s}$ as example. 


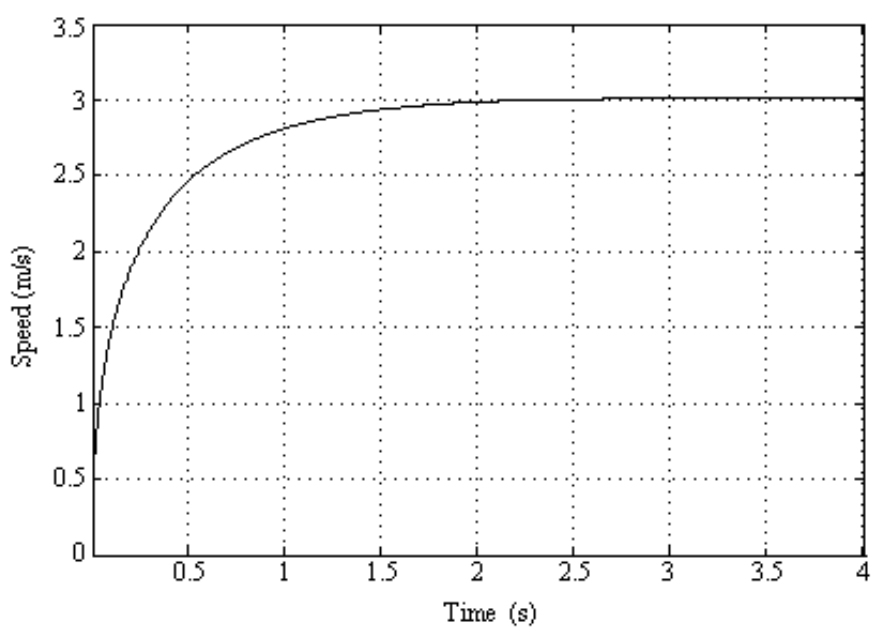

Fig. 8 Speed curve during start-up.

\section{CONCLUSION}

This paper presents a design and analysis of a HTS levitator with a HTS LSM driving system for the technology verification. The HTS can specially function in the design as magnets for levitation, guidance, and driving. Different computing methods are used for its performance analysis. Results show that the HTS levitated system with proposed design and its driving scheme can be effectively operated and controlled, and the simulated results show that the designed HTS driving system is capable of driving the prototype at the desired speed. HTS well developed allows the levitated mobiles built in various scales to suit different applications. Both electromagnetic properties and operational characteristics of the critical element HTS in this technology are suitable to practical applications with the superior performance which can not be achieved using other techniques to date.

\section{ACKNOWLEDGMENT}

The authors would thank Mr. J. Wen for providing assistance to the work.

\section{REFERENCES}

[1] J.G. Bednorz, and K.A. Müller, "Possible high $T_{C}$ superconductivity in the Ba-La-Cu-O system," Zeitschrift fur Physik B-Condensed Matter, vol. 64, no. 2, pp. 189-193, 1986.

[2] J.X. Jin, L.H. Zheng, R.P. Zhao, J. Zhang, L. Jiang, J.H. Chen, Y.L. Jiang, and H.Y. Zhang, "Practical applications of high temperature superconductors,” Nature Sciences, vol. 1, no. 1, pp. 48-79, December 2006.

[3] T.M. Silver, S.X. Dou, and J.X. Jin, "Applications of high temperature superconductors,” Europhysics News, vol. 32, no. 3, pp. 82-86, May/June 2001.

[4] J.X. Jin, and L.H. Zheng, "Development and applications of high temperature superconducting material," Journal of University of Electronic Science and Technology of China, vol. 35, no. 4, pp. 612627, September 2006.

[5] J.X. Jin, "Twenty years of high $T_{c}$ superconductivity," Nature Sciences, vol. 1, no. 1, pp. 1-16, December 2006.
[6] L.H. Zheng, J.X. Jin, Y.G. Guo, H.Y. Lu, and J.G. Zhu, "Technology and development of high temperature superconducting linear motors," Proceedings of Australasian Universities Power Engineering Conference, TS06.3/1-4, Melbourne, Australia, December 2006.

[7] J.X. Jin, S.X. Dou, H.K. Liu, R. Neale, N. Attwood, G. Grigg, T. Reading, and T. Beales, "A high gradient magnetic separator fabricated using Bi-2223/Ag HTS tapes," IEEE Transactions on Applied Superconductivity, vol. 9, no. 2, pp. 394-397, June 1999.

[8] J.X. Jin, S.X. Dou, F. Darmann, M. Apperley, and T. Beales, "Development of an HTS inductor for an electronic high voltage generator,” Physica C, vol. 341-348, pp. 1601-1602, November 2000.

[9] J.X. Jin, S.X. Dou, H.K. Liu, and C. Grantham, "High voltage generation with a high $\mathrm{T}_{\mathrm{c}}$ superconducting resonant circuit," IEEE Transactions on Applied Superconductivity, vol. 7, no. 2, pp. 881-884, June 1997.

[10] L.H. Zheng, J.X. Jin, Y.G. Guo, and J.G. Zhu, "Technical and theoretical analysis of hts machines and their development," Nature Sciences, vol. 1, no. 1, pp. 33-41, December 12, 2006.

[11] R. Muramatsu, S. Sadakata, M. Tsuda, and A. Ishiyama, "Trial production and experiments of linear actuator with HTS bulk secondary," IEEE Transactions on Applied Superconductivity, vol. 11, no. 1, pp. 1976-1979, 2001.

[12] A. Takahashi, H. Ueda, and A. Ishiyama, "Trial production and experiment of linear synchronous actuator with field-cooled HTS bulk secondary," IEEE Transactions on Applied Superconductivity, vol. 13, no. 2, pp. 2251-2254, 2003.

[13] A. Sato, H. Ueda and, and A. Ishiyama, "Operational characteristics of linear synchronous actuator with field-cooled HTS bulk secondary," IEEE Transactions on Applied Superconductivity, vol. 15, no. 2, pp. 2234-2237, 2005.

[14] G. Stumberger, M.T. Aydemir, and A.L. Thomas, "Design of a linear bulk superconductor magnet synchronous motor for electromagnetic aircraft launch systems," IEEE Transactions on Applied Superconductivity, vol. 14, no. 1, pp. 54-62, 2004.

[15] W.S. Kim, S.Y. Jung, H.Y. Choi, H.K. Jung, J.H. Kim, and S.Y. Hahn, "Development of a superconducting linear synchronous motor," IEEE Transactions on Applied Superconductivity, vol. 22, no. 1, pp. 842845, 2002.

[16] F. Hellman, E.M. Gyorgy, D.W. Johnson Jr., H.M. O’Bryan, and R.C. Sherwood, "Levitation of a magnet over a flat type II superconductor," Journal of Applied Physics, vol. 63, pp. 447-450, January 1988.

[17] J.X. Jin, "High $T_{C}$ superconductor theoretical models and electromagnetic flux characteristics," Journal of Electronic Science and Technology of China, vol. 4, no. 3, pp. 202-208, September 2006.

[18] B. Li, and J.X. Jin, "Theoretical models and analysis of HTS and magnetic field interaction,” Nature Sciences, vol. 1, no. 1, pp. 42-47, December 12, 2006.

[19] T. Takao, A. Niiro, S. Suzuki, M. Hashimoto, H. Kamijo, and H. Fujimoto, "Experimental and numerical analysis of lift force in magnetic levitation system," IEEE Transactions on Applied Superconductivity, vol. 15, no. 2, pp. 21281-2284, June 2005.

[20] G.W. Mclean, "Review of recent progress of linear motors," IEE Proceedings - Part B, vol. 135, no. 6, pp. 380-416, November 1988.

[21] Y.G. Guo, J.G. Zhu, and H.Y. Lu, "Accurate determination of parameters of a claw pole motor with SMC stator core by finite element magnetic field analysis,” IEE Proceedings - Electri. Power Appl., vol. 153, no. 4, pp. 568-574, July 2006.

[22] O.A. Mohammed, S. Liu, and Z. Liu, "A phase variable model of brushless dc motors based on finite element analysis and its coupling with external circuits," IEEE Transactions on Magnet, vol. 41, no. 5, pp. 1576-1579, May 2005.

[23] Y.G. Guo, J.G. Zhu, J.X. Chen, and J.X. Jin, "Performance analysis of a permanent magnet claw pole SMC motor with brushless dc control scheme," in Proc. International Power Electronics and Motion Control Conference, Shanghai, China, pp. 11.8/1-5, August 13-16, 2006. 